Journal of Contemporary Educational Research

Research Article

\title{
The Influence of Contemporary Dance, Used in the Choreographic Process of Chinese Mongolian dance
}

\author{
Xiaoyu $\mathrm{Mu}^{*}$ \\ Shandong University, Weihai 264200, Shandong Province, China
}

\begin{abstract}
China is a country that has 55 nationalities. The Chinese folk dance is a dance that also has 55 varieties of folk dances. Chinese Mongolian dance is one of those folk dances. The Chinese Mongolian dance mentioned in this paper can also be interpreted as an academically trained Chinese Mongolian dance.
\end{abstract}

Publication date: August, 2020

Publication online: 31 August, 2020

*Corresponding author: Xiaoyu Mu, xiaoyu.mu14@bathspa.ac.uk

Nowadays, with the spread of western culture in China, Chinese aesthetics have changed a lot for example in relation to art, pace of life, thought patterns, mode of emotion and so on. Therefore, a lot of people in China are more interested in the diversity of dance. The first reason for this is that diverse dance forms can express different people's feelings and emotions (Darlene O'Cadiz, 2013). The second one is that diversity of dance can bring different visual effects to the audience. As a result, innovative choreographers continuously reform Mongolian dance incorporating different dance elements into their own Chinese Mongolian dance work. For example, Chinese Mongolian dance mixes Contemporary dance elements and so on. In the course of development national folk dance has its own characteristics, but folk dance also actively absorbs and learns from the different kinds of dance features and advantages, especially from Western Contemporary dance and western Contemporary dance training (Feng shuang bai, 2002). Nowadays, Mongolian dance mixed with Contemporary dance element has been implemented in China. However, mixing Contemporary dance elements with Chinese Mongolian dance may be difficult because Contemporary dance in terms of dance movement, rhythm, narrative and so on, have different characteristics to Chinese Mongolian dance. This essay will have five parts. The first part is asking what we mean by "Chinese Mongo- lian dance" and "Contemporary dance". Secondly, how Contemporary dance elements can be used in Chinese Mongolian dance. The third, whether using Contemporary dance elements in Chinese Mongolian dance has any disadvantages. In the fourth part, whether using Contemporary dance elements in Chinese Mongolian dance has any advantages.

The Chinese folk dance is a symbol of nationality. The Chinese folk dance also represents the traditional Chinese culture that has distinct characteristics and aesthetic. China has fifty-five officially recognized ethnic minorities. Each nationality, each region and each type of dance carries it is own folk dance forms with unique features and flavors. Folk dances directly reflect the lifestyles and customs of a people (He yuan, 2014). Chinese ethnic and folk dance represents a vast and varied assortment of traditional dance styles. Chinese ethnic dance generally refers to the dance forms passed down among the many minority ethnic groups within China. Their distinct ways of life reflect discrepancies in local topography, climate, and religious tradition. Therefore, each ethnic minority has their own dance style and features because they live in different areas, have different living habits and different cultures. Over China's long history, the movements and style of each region's traditional ethnic and folk dances have undergone gradual codification to the point where today they can be widely recognized in the dance world (Jia zuo guang, 1998).

Mongolian dance is one of the 55 ethnic folk dances that have unique dance form, costumes, and props. The Chinese Mongolian dance creations represent real lives in their story plots and folk customs (Jia zuo guang, 1998). In the dance form the Mongolian dance, most of the dance movements are neat and most dancers will do the same dance moves. Secondly, Chinese Mongolian dance has less the movements shape contrast (sdcfde San Diego, 2013 ). The third element of Mon- 
golian dance is that it has a large range of motion and the movements are flexible and free. Because Mongolia has a vast land and there is a lot of grassland, animal husbandry is Mongolia's leading industry. It also was c known as a nationality on horseback by Chinese people (China Highlights, 2016). Since Mongolia has vast prairie Mongolian dance has a lot of imitation animal movements, such as those of geese, and horses. As a result, Mongolian dance has a lot of movements using arms and shoulders, because these arm movements are like people riding a horse (redianzongyi, 2014) and the movements of flying geese (Amy45487, 2015).

In relation to Mongolian dance props, Mongolia has a lot of dance work with props such as chopsticks, bowls, and a small sake wine cup (sdcfde San Diego, 2013). In the Mongolian steppes, when the traditional festival comes, the people, in order to express their joy, will sing, and will pick up small sake cups, bowls, and chopsticks while dancing and singing. As a result, this tradition created unique Mongolian style of dance. Before the emergence of Contemporary Chinese Mongolian dance, Chinese Mongolian dance was created mainly from folklore folk tales, and national symbols. This creation is restricted because the Chinese folk dance moves in a single and changes little, movements is regularity, and the Chinese Mongolian dance works mainly reflects in the emotion and ethnic costumes and props (Wang Ke fen, 1985).

Unlike the Chinese Mongolian dance, the Contemporary dance is a form of dance which focuses on the serious expression of inner emotions, using a free-flowing, interpretive style, instead of following the strict rules characteristic of many dance disciplines (Anderson. J, 1992). Contemporary dance emerged in the second half of the twentieth century as a response to the rigors of classical ballet. First, Contemporary dance movements were more relaxed and unrestrained in the pursuit of freedom, because this broke the strict dance movements of classical ballet. In the second place, Contemporary dance has many variations in relation to shape and space, A lot of Contemporary dance work shows the beauty of asymmetry: "Contemporary dance movements broke the classical ballet's strict movement vocabulary and defined by a re-thinking of the body and physicality in relationship to space, time and gravity ; and by a cross - disciplinary and collaborative approach with disciplines such as philosophy, cultural theory, experimental music, visual arts and multimedia" (Pierce, 2015). Fourth, in relation to the dance costumes, Contemporary dance costumes are comfortable and more life dance costumes. This is seen, for example, In the 'Rice' that was created by huai min Lin who is a famous choreographer from Taiwan (ravennafestival, 2010). This dance tells the story of the life cycle of rice. It has flexible movements, diversification of shape and casual and comfortable dance costumes. The movements, the dancer always repeat the same action. Although, the dancer's upper body movements is different, their legs movement is the same. This Contemporary dance works movements are simple and most are action rhythm of change. Fluent movement has been is the symbol of the Contemporary dance form. The Contemporary dance work 'Rice' also has a lot of fluent movements. It also has a lot of the movement amplitude of variation. At the Contemporary dance work beginning, there are have no music that seems like the seeds lay quiet in the land. After with the dance movements amplitude of variation, there are have the vocal accompaniment. Contemporary dance performance always the male and female perform together (Feng shuangbai, 2002). The Contemporary dance work 'Rice' also is the male and female perform together. This Contemporary dance work also has a lot of the shape contrast, at the same time these dancers will do the different movements. In the middle of this Contemporary dance work, the movement and action rhythm have changed. The dancer will do the different action rhythm at the same time(Zhang xin lan, 2015). Because this Contemporary dance work tells the story of the life cycle of rice. Therefore, a lot of movements are circle movements and also have a lot of ground rolling movements. In the Contemporary dance's dance costumes, unlike Chinese Mongolian dance costumes, this Contemporary dance work the female also wear the simple and comfortable dress, and the male wear the T-shirt.

In China, there are a lot of choreographers who incorporate Contemporary dance elements into Chinese Mongolian dance, but how to blend these Contemporary dance elements has become a focus. First, the most important step is for dancers to gain Contemporary dance training. In China, there are many dance schools that offer Contemporary dance as a basic training course ( xiang xu, 2013). Because the Contemporary dance training focuses on balance and coordination. Since, the Contemporary dance movements are relaxed and the body therefore needs increased levels of balance and coordination. Secondly, Contemporary dance develops the student's joint flexibility because it uses muscles in a different way to traditional dance. Therefore, holding muscles in a stretched position for a prolonged amount of time causes the muscle fibers to become accustomed to the new length, therefore increasing flexibility (Bompa, 1999 ).

Only if dancers accept the Contemporary dance train- 
ing can they make the body more flexible, and improve balance and coordination, thereby being ready to interpret and perform the choreographer's dance that combines Contemporary dance and the Chinese Mongolian dance (Bompa, 1999). The Second, in the process of choreographing, retaining the national characteristics is also very important. In the creation of Contemporary Mongolian dance, clear national attributes must first be represented, and then Contemporary dance elements can be added into Chinese Mongolian dance (Gao du, 2014). The Chinese folk dance "Leave" is a Chinese Contemporary Mongolian dance and male dance work (linling chen, 2016). This Mongolian dance work is different to previous Mongolian dance work because the choreographers added some Contemporary dance elements and the dancers who took Contemporary dance training. This Contemporary Mongolian dance work's background is based around the Mongolian grassland, thus reflecting traditional Mongolian lives. It focuses on two young boys. One of the boys wants to leave the grassland and he wants to see the outside world. The other boy hopes his friend will stay in the grassland. The movements of this dance work, not only use the Mongolian dance normative actions like arm and shoulder movements, but also use a lot of freer Contemporary dance rolling movements. There are also some movements where Contemporary dance rolling movements combine with Mongolian dance arm movements. The third, this Mongolian dance work has a vast number of Contemporary dance movements that first space and the second space contrast to the action. One of the dancers stands and does some movements, while another one dancer does ground movements and Contemporary dance rolling movements. In relation to emotional expression, in addition to retaining the traditional, this Mongolian dance work represents the emotional expression of Contemporary dance where the dancer's performance represents a state of mind rather than simply telling a story and representing folk customs (Gao du, 2014).

"Chinese Mongolian dance combined with elements of Contemporary dance dances make great progress" (Gao du, 2014). Therefore, this great progress could be divided into several aspects. Firstly, One benefit of combining Contemporary dance and Chinese Mongolian dance that these two kinds of dance can be complementary. In the Mongolian dance work elements in the appropriate integration of Contemporary dance, Contemporary dance aesthetic value can be given to achieve artistic innovation (Li Bei da, 2006). It also has been proven in the dance practice for example the Chinese Mongolian dance work "Leave" because it won numerous awards in China and It was retained as a classic work in Minzu University of China. Secondly, folk dance and Contemporary dance are two kinds of important dance in China (Feng shang bai, 2002). Combination of the two types of dance elements beneficial to improve the two types of dance because in dance development process, dance also need to satisfy the audience's vision, comply with the development of society. Therefore, combine with Contemporary dance elements into folk dance have an advantage that different kinds of dance lessons and inheritance, continue to made breakthroughs in style, diversification, many changes, personalized trends, and mutual promotion and development. Thirdly, Folk dance and Contemporary dance has a different style and aesthetic, folk dance elements into Contemporary dance which beneficial Identify the nationality of Contemporary dance. The Contemporary dance elements into folk dance benefit the development of new folk dance performance style. The fifth, Chinese folk dance combined with Contemporary dance elements benefits A lot of ways for example, in the shape, body language, and thought have a specific embodiment. In shape, the folk dance choreographer is pursuit of asymmetrical shape rather than the symmetrical modelling. In body language, to abandon the traditional mechanized movement, and change the use of space to create visual effects, for example the Contemporary dance rolling movements into the traditional Mongolian dance. In terms of ideology, The dancers gave up the Chinese Mongolian dance work that the expression of a collective focus on the inner spirit, repressed personal feeling. In contrast, the dancers expression Contemporary dance and more free, open, and the performance of personal emotions.

The Chinese Mongolian dance combined with Contemporary dance elements also may have an impact on folk dance cultural heritage. Therefore, it may make the Mongolian dance work become Contemporary ized (Feng shuang bai, 2002). Because the Chinese Mongolian dance and Contemporary dance have different cultural backgrounds. China is country that has a history of five thousand years. In relation to culture and art, it has formed its own unique form and there is a profound ideological feudal culture. On the contrary, Contemporary dance is avant-garde art that this was followed by the successive movements of Contemporary art, and the term avant-garde is more or less synonymous with Contemporary (TATE, 2016). Contemporary dance is novel dance form with no complicated plot, scenery, music, irregular or dancer can create their own music or sound. The Chinese Mongolian dance form is traditional that using their 
own the traditional music, clothing, and props. As a result, for most people who are feudal it is difficult to accept the novel Contemporary dance elements into traditional Chinese Mongolian dance. In the beginning, Contemporary dance elements in traditional Chinese Mongolian dance resulted in failure (Jia zuo guang, 1998). First, before the creation of dance works the choreographer does not determine national property. For the Chinese Mongolian dance works the most important is determine national property (Jia zuo guang, 1998) because have no national property the Chinese Mongolian dance works may become Contemporary ization. As a result, the dance works that have no national property may become Contemporary dance work rather than the Contemporary Mongolian dance works. The second, when some choreographers create, they are using Contemporary dance movements that have no change into the Contemporary Mongolian dance works. Therefore, this create also may make the Contemporary Mongolian dance works become Contemporary ization. Not all of Contemporary dance movements are suitable for incorporating in Mongolian dance works. If the Mongolian dance works add inappropriate Contemporary dance movements or have no changes Contemporary dance movements may make the movements lack the style of Mongolian dance. The third, dancers only accept Contemporary dance training for a long time that the dancers will automatically generate the memory of the body and the formation of Contemporary dance movements. But Chinese choreographers find a way to combine two kinds of dance immediately. The way is change in Contemporary dance elements and make Contemporary dance elements are more easily into the Chinese Mongolian dance work. For example, the Contemporary Mongolian dance work "leave," the choreographers make rolling combined with Mongolian arm movements (Lin ling chen, 2016).

In conclusion, although using the western Contemporary dance elements in Chinese Mongolian dance has some advantages. For example, make the Chinese Mongolian dance work more intact that change the Chinese Mongolian dance single movements and shape thereby give the audience different visual effects. It still has some disadvantages that may have culture shock and may make the Chinese Mongolian dance work become Contemporary ization. If reduce these culture shocks, the Chinese Mongolian dance work would be not become Contemporary ization and could make the Chinese Mongolian dance work more complete. The choreographers need make Contemporary dance elements into Chinese Mongolian dance using Contemporary dance elements that suitable for the Mongolian dance moves rather than the Contemporary dance elements that have no change into Contemporary Mongolian dance. Secondly, even is Contemporary Mongolian dance work, it also needs to have the Mongolian dance elements and background. The third, the dancers not only need the Contemporary dance training but also need the Chinese Mongolian dance training. As a result, using the western Contemporary dance elements in the Chinese Mongolian dance work may have some challenges, but if avoid these the disadvantages. The Chinese Contemporary Mongolian dance become more complete.

\section{References}

[1] Bompa T. (1999) Theory and Methodology of Training China Highlights [online] Available from: http://www.chinahighlights. com/travelguide/nationality/ Accessed by 15 Jan 2016.

[2] Darlene O'Cadiz. (2013) Dance and Cultural Diversity.

[3] Feng Shangbai. (2002) A history of Chinese dance.

[4] Gong Shiershao. (2015) [online] Available from:https://www. youtube.com/watch? $=5$ cSNcwkftpk Accessed by $10 \mathrm{Jan}$ 2016.

[5] Gao Du. (2014) Introduction to Chinese Folk Dance.

[6] He Yuan. (2014) History of Chinese ancient dance.

[7] Jia Zuoguang. (1998) History of Chinese folk dance.

[8] Lin Lingchen. (2014) [online] Available from:https://www. youtube.com/watch?v=b4UNqHJpnQ4 Accessed by $22 \mathrm{Dec}$ 2015.

[9] Pierce, Julianne.(2015) Perspectives on contemporary dance.

[10] Ravennafestival. (2010) [online] Available from: https://www. youtube.com/watch? $\mathrm{v}=1 \mathrm{aXD} 14 \mathrm{t}-6 \mathrm{QY}$ Accessed by $15 \mathrm{Jan}$ 2016.

[11] Sdcfde San Diego. (2013) [online] Available from: https:// www.youtube.com/watch?v=RqYkvFgdBs8 Accessed by 15 Jan 2016

[12] Wang Kefen. (1985) The History of Chinese Dance.

[13] Wu Yunming. (2010) Chinese Primitive Dance Culture.

[14] Xiang Xu. (2013) Basic Training for Contemporary Dance [online] Available from: https://www.youtube.com/watch?v=wz ayNDjHJU Accessed by 11 Jan 2016

[15] Zhang Xinlan. (2015) [online] Available from: https://www. youtube.com/watch?v=dpiP2YhsnTc Accessed by 30 Dec 2015. 